Bull. Austral. Math. Soc.

VOL. 66 (2002) [25-31]

\title{
A NOTE ON TENSOR PRODUCTS OF REFLEXIVE ALGEBRAS
}

\section{ZHE DONG}

In this short note, we obtain a concrete description of rank-one operators in $\operatorname{Alg}\left(\mathcal{L}_{1} \otimes\right.$ $\left.\cdots \otimes \mathcal{L}_{n}\right)$. Based on this characterisation, we give a simple proof of the tensor product formula:

$$
\operatorname{Alg}\left(\mathcal{L}_{1} \otimes \cdots \otimes \mathcal{L}_{n}\right)=\operatorname{Alg} \mathcal{L}_{1} \otimes_{w} \cdots \otimes_{w} \operatorname{Alg} \mathcal{L}_{n}
$$

if $\operatorname{Alg}\left(\mathcal{L}_{1} \otimes \cdots \otimes \mathcal{L}_{n}\right)$ is weakly generated by rank-one operators in itself and $\mathcal{L}_{i}(i$ $=1, \ldots, n)$ are subspace lattices.

\section{INTRODUCTION}

One of the central results in the theory of tensor products of von Neumann algebras is Tomita's commutation formula:

$$
\mathcal{M}^{\prime} \otimes_{w} \mathcal{N}^{\prime}=\left(\mathcal{M} \otimes_{w} \mathcal{N}\right)^{\prime},
$$

where $\mathcal{M}$ and $\mathcal{N}$ are von Neurnann algebras. It was observed in [1] that if we let $\mathcal{L}_{1}$ and $\mathcal{L}_{2}$ denote the projection lattices of $\mathcal{M}$ and $\mathcal{N}$ respectively, then (1) can be rewritten as

$$
\operatorname{Alg} \mathcal{L}_{1} \otimes_{w} \operatorname{Alg} \mathcal{L}_{2}=\operatorname{AIg}\left(\mathcal{L}_{1} \otimes \mathcal{L}_{2}\right)
$$

This version of Tomita's theorem makes sense for any pair of reflexive algebras $\operatorname{Alg} \mathcal{L}_{1}$ and $\operatorname{Alg} \mathcal{L}_{2}$. It remains a deep open question whether the tensor product formula (2) is valid for general reflexive algebras, or even general commutative subspace lattice algebras. However, (2) has been proved in a number of special cases $([\mathbf{1}, \mathbf{2}, \mathbf{3}, \mathbf{4}])$. In particular, it is known that if $\mathcal{L}_{1}$ is a commutative subspace lattice that is either completely distributive ([4]) or finite width ([2]), then (2) is valid for $\mathcal{L}_{1}$ and any subspace lattice $\mathcal{L}_{2}$. The main purpose of this paper is to study the $n$-fold tensor product formula of reflexive algebras. The technique employed in this note is simple and different from the other papers about tensor products. We use rank-one operators to investigate tensor products and the technique shows its power in this note.

Let us introduce some notation and terminology. Throughout, $\mathcal{H}$ represents a complex separable Hilbert space, $\mathcal{B}(\mathcal{H})$ the algebra of bounded operators on $\mathcal{H}$. A sublattice

Received 22nd November, 2001

Copyright Clearance Centre, Inc. Serial-fee code: 0004-9727/02 \$A2.00+0.00. 
$\mathcal{L}$ of the projection lattice of $\mathcal{B}(\mathcal{H})$ is said to be a subspace lattice if it contains 0 and $I$ and is strongly closed, where we identify projections with their ranges. If the elements of $\mathcal{L}$ pairwise commute, $\mathcal{L}$ is a commutative subspace lattice. A nest is a totally ordered subspace lattice. If $\mathcal{L}$ is a subspace lattice, $\operatorname{Alg} \mathcal{L}$ denotes the set of operators in $\mathcal{B}(\mathcal{H})$ that leave the elements of $\mathcal{L}$ invariant. If $\mathcal{L}$ is a commutative subspace lattice, $\operatorname{Alg} \mathcal{L}$ is said to be a commutative subspace lattice algebra. If $\mathcal{L}$ is a nest, $\operatorname{Alg} \mathcal{L}$ is said to be a nest algebra.

If $\mathcal{A}$ is a subset of $\mathcal{B}(\mathcal{H})$ then Lat $\mathcal{A}$, the set of projections left invariant by each element of $\mathcal{A}$, is a subspace lattice. A subalgebra $\mathcal{A}$ of $\mathcal{B}(\mathcal{H})$ is reflexive if $\mathcal{A}=\operatorname{Alg}$ Lat $\mathcal{A}$. The reflexive algebras are precisely the algebras of the form $\operatorname{Alg} \mathcal{L}$, where $\mathcal{L}$ is a subspace lattice. If $\mathcal{L}_{i} \subseteq \mathcal{B}\left(\mathcal{H}_{i}\right)(i=1, \ldots, n)$ are subspace lattices, $\mathcal{L}_{1} \otimes \cdots \otimes \mathcal{L}_{n}$ is the subspace lattice in $\mathcal{B}\left(\mathcal{H}_{1} \otimes \cdots \otimes \mathcal{H}_{n}\right)$ generated by $\left\{L_{1} \otimes \cdots \otimes L_{n}: L_{i} \in \mathcal{L}_{i}, i=1, \ldots, n\right\}$. If $\mathcal{S}_{i} \subseteq \mathcal{B}\left(\mathcal{H}_{i}\right)(i=1, \ldots, n)$ are subspaces, then $\mathcal{S}_{1} \otimes \cdots \otimes \mathcal{S}_{n}$ denotes the linear span of $\left\{S_{1} \otimes \cdots \otimes S_{n}: S_{i} \in \mathcal{S}_{i}\right\} ; \mathcal{S}_{1} \otimes_{w} \cdots \otimes_{w} \mathcal{S}_{n}$ denotes the weak closure of $\mathcal{S}_{1} \otimes \cdots \otimes \mathcal{S}_{n}$ in $\mathcal{B}\left(\mathcal{H}_{1} \otimes \cdots \otimes \mathcal{H}_{n}\right)$.

\section{TENSOR PRODUCTS OF REFLEXIVE ALGEBRAS}

For $x, y \in \mathcal{H}$, the operator $x y^{*}$ is defined by the equation

$$
\left(x y^{*}\right)(z)=\langle z, y\rangle x, \quad \text { for all } \quad z \in \mathcal{H} \text {. }
$$

If $\mathcal{L}$ is a subspace lattice and $L \in \mathcal{L}$, we write $L_{-}$for the projection $\vee\{E \in \mathcal{L}$ : $L \notin E\}$. The following result of Longstaff $[6]$ is essential.

LEMma 1. Let $\mathcal{L}$ be a subspace lattice. Then $x y^{*} \in \operatorname{Alg} \mathcal{L}$ if and only if there is an element $L \in \mathcal{L}$ such that $x \in L$ and $y \in L_{-}^{\perp}$.

Let $\mathcal{L}_{i} \subseteq \mathcal{B}\left(\mathcal{H}_{i}\right)(i=1, \ldots, n)$ be subspace lattices. For $1 \leqslant j \leqslant n$, let $I_{1} \otimes \cdots \otimes \mathcal{L}_{j}$ $\otimes \cdots \otimes I_{n}=\left\{I_{1} \otimes \cdots \otimes L_{j} \otimes \cdots \otimes I_{n}: L_{j} \in \mathcal{L}_{j}\right\}$; certainly, it is a subspace lattice.

LEMmA 2 . Let $\mathcal{L}_{j} \subseteq \mathcal{B}\left(\mathcal{H}_{i}\right)(1 \leqslant j \leqslant n)$ be subspace lattices. Suppose that $N_{j} \in \mathcal{L}_{j}$, then

$$
\left(I_{1} \otimes \cdots \otimes N_{j} \otimes \cdots \otimes I_{n}\right)_{-}=I_{1} \otimes \cdots \otimes N_{j-} \otimes \cdots \otimes I_{n}
$$

and

$$
\left(I_{1} \otimes \cdots \otimes N_{j} \otimes \cdots \otimes I_{n}\right)_{-}^{\perp}=I_{1} \otimes \cdots \otimes N_{j-}^{\perp} \otimes \cdots \otimes I_{n}
$$

in $I_{1} \otimes \cdots \otimes \mathcal{L}_{j} \otimes \cdots \otimes I_{n}$.

PROOF: We first show that $I_{1} \otimes \cdots \otimes N_{j} \otimes \cdots \otimes I_{n} \leqslant I_{1} \otimes \cdots \otimes L_{j} \otimes \cdots \otimes I_{n}$ if and only if $N_{j} \leqslant L_{j}$. For the forward implication choose unit vectors $x_{i} \in \mathcal{H}_{i}(i \neq j)$. For any $x_{j} \in \mathcal{H}_{j}$,

$$
\begin{aligned}
0 & \leqslant\left\langle\left(I_{1} \otimes \cdots \otimes\left(L_{j}-N_{j}\right) \otimes \cdots \otimes I_{n}\right)\left(x_{1} \otimes \cdots \otimes x_{n}\right), x_{1} \otimes \cdots \otimes x_{n}\right\rangle \\
& =\left\langle x_{1} \otimes \cdots \otimes\left(L_{j}-N_{j}\right) x_{j} \otimes \cdots \otimes x_{n}, x_{1} \otimes \cdots \otimes x_{n}\right\rangle \\
& =\left\langle\left(L_{j}-N_{j}\right) x_{j}, x_{j}\right\rangle .
\end{aligned}
$$


So $N_{j} \leqslant L_{j}$. The converse implication is also natural. Thus $I_{1} \otimes \cdots \otimes N_{j} \otimes \cdots \otimes I_{n}$ $\not I_{1} \otimes \cdots \otimes L_{j} \otimes \cdots \otimes I_{n}$ if and only if $N_{j} \nless L_{j}$. Hence

$$
\begin{aligned}
\left(I_{1}\right. & \otimes \\
& \left.\cdots \otimes N_{j} \otimes \cdots \otimes I_{n}\right)_{-} \\
& =\vee\left\{I_{1} \otimes \cdots \otimes L_{j} \otimes \cdots \otimes I_{n}: I_{1} \otimes \cdots \otimes N_{j} \otimes \cdots \otimes I_{n} \notin I_{1} \otimes \cdots \otimes L_{j} \otimes \cdots \otimes I_{n}\right\} \\
& =\vee\left\{I_{1} \otimes \cdots \otimes L_{j} \otimes \cdots \otimes I_{n}: N_{j} \notin L_{j}\right\} \\
& =I_{1} \otimes \cdots \otimes\left(\vee\left\{L_{j}: N_{j} \nless L_{j}\right\}\right) \otimes \cdots \otimes I_{n} \\
& =I_{1} \otimes \cdots \otimes N_{j-} \otimes \cdots \otimes I_{n}
\end{aligned}
$$

(The proof of the third equality is routine). Since

$$
\left(I_{1} \otimes \cdots \otimes N_{j-}^{1} \otimes \cdots \otimes I_{n}\right)\left(I_{1} \otimes \cdots \otimes N_{j-} \otimes \cdots \otimes I_{n}\right)=0 \text {, }
$$

we have

$$
I_{1} \otimes \cdots \otimes N_{j-}^{\perp} \otimes \cdots \otimes I_{n} \leqslant\left(I_{1} \otimes \cdots \otimes N_{j-} \otimes \cdots \otimes I_{n}\right)^{\perp} .
$$

If $\left(I_{1} \otimes \cdots \otimes N_{j-} \otimes \cdots \otimes I_{n}\right)^{\perp} \neq I_{1} \otimes \cdots \otimes N_{j-}^{\perp} \otimes \cdots \otimes I_{n}$, we can choose a non-zero vector $z \in\left(I_{1} \otimes \cdots \otimes N_{j-} \otimes \cdots \otimes I_{n}\right)^{\perp} \ominus\left(I_{1} \otimes \cdots \otimes N_{j-}^{\perp} \otimes \cdots \otimes I_{n}\right)$. Thus

$$
\begin{aligned}
z & =\left(I_{1} \otimes \cdots \otimes I_{j} \otimes \cdots \otimes I_{n}\right) z \\
& =\left(I_{1} \otimes \cdots \otimes N_{j-} \otimes \cdots \otimes I_{n}\right) z+\left(I_{1} \otimes \cdots \otimes N_{j-}^{\perp} \otimes \cdots \otimes I_{n}\right) z \\
& =0
\end{aligned}
$$

This is a contradiction. So

$$
\begin{aligned}
\left(I_{1} \otimes \cdots \otimes N_{j} \otimes \cdots \otimes I_{n}\right)_{-}^{\perp} & =\left(I_{1} \otimes \cdots \otimes N_{j-} \otimes \cdots \otimes I_{n}\right)^{\perp} \\
& =I_{1} \otimes \cdots \otimes N_{j-}^{\perp} \otimes \cdots \otimes I_{n} .
\end{aligned}
$$

LEMma 3. Let $\mathcal{L}_{i} \subseteq \mathcal{B}\left(\mathcal{H}_{i}\right)(i=1, \ldots, n)$ be subspace lattices. Then a rankone operator $x y^{*} \in \operatorname{Alg}\left(\mathcal{L}_{1} \otimes \cdots \otimes \mathcal{L}_{n}\right)$ if and only if there exist $N_{i} \in \mathcal{L}_{i}$ such that $x \in N_{1} \otimes \cdots \otimes N_{n}$ and $y \in N_{1-}^{\perp} \otimes \cdots \otimes N_{n-}^{\perp}$.

PRoOF: Set $\mathcal{F}_{i}=I_{1} \otimes \cdots \otimes \mathcal{L}_{i} \otimes \cdots \otimes I_{n}$. Thus

$$
\mathcal{L}_{1} \otimes \cdots \otimes \mathcal{L}_{n}=\mathcal{F}_{1} \vee \cdots \vee \mathcal{F}_{n}
$$

and

$$
\operatorname{Alg}\left(\mathcal{L}_{1} \otimes \cdots \otimes \mathcal{L}_{n}\right)=\left(\operatorname{Alg} \mathcal{F}_{1}\right) \cap \cdots \cap\left(\operatorname{Alg} \mathcal{F}_{n}\right)
$$

Now suppose that $x y^{*} \in \operatorname{Alg}\left(\mathcal{L}_{1} \otimes \cdots \otimes \mathcal{L}_{n}\right)$. Since $\mathcal{L}_{1} \otimes \cdots \otimes \mathcal{L}_{n} \supseteq \mathcal{F}_{i}, \operatorname{Alg}\left(\mathcal{L}_{1}\right.$ $\left.\otimes \cdots \otimes \mathcal{L}_{n}\right) \subseteq \operatorname{Alg} \mathcal{F}_{i}$. Thus $x y^{*} \in \operatorname{Alg} \mathcal{F}_{i}$ and, by the definition of $\mathcal{F}_{i}$ and Lemma 1 and Lemma 2, there is an element $N_{i} \in \mathcal{L}_{i}$ such that $x \in I_{1} \otimes \cdots \otimes N_{i} \otimes \cdots \otimes I_{n}$ and $y \in\left(I_{1} \otimes \cdots \otimes N_{i} \otimes \cdots \otimes I_{n}\right)_{-}^{\perp}=I_{1} \otimes \cdots \otimes N_{i-}^{\perp} \otimes \cdots \otimes I_{n}$. This is valid for each $i=1, \ldots, n$, whence

$$
x \in N_{1} \otimes \cdots \otimes N_{n} \quad \text { and } \quad y \in N_{1-}^{\perp} \otimes \cdots \otimes N_{n-}^{\perp}
$$


For the converse, if $x \in N_{1} \otimes \cdots \otimes N_{n}$ and $y \in N_{1-}^{\perp} \otimes \cdots \otimes N_{n-}^{\perp}$ then, in particular, $x \in I_{1} \otimes \cdots \otimes N_{i} \otimes \cdots \otimes I_{n}$ and $y \in I_{1} \otimes \cdots \otimes N_{i-}^{1} \otimes \cdots \otimes I_{n}$. Lemma 1 and Lemma 2 imply that $x y^{*} \in \operatorname{Alg} \mathcal{F}_{i}$, for each $i$. Hence

$$
x y^{*} \in \bigcap_{i=1}^{n} \operatorname{Alg} \mathcal{F}_{i}=\operatorname{Alg}\left(\mathcal{L}_{1} \otimes \cdots \otimes \mathcal{L}_{n}\right) .
$$

Proposition 4. Let $\mathcal{L}_{i} \subseteq \mathcal{B}\left(\mathcal{H}_{i}\right)(i=1, \ldots, n)$ be subspace lattices. If $L$ $\in \mathcal{L}_{1} \otimes \cdots \otimes \mathcal{L}_{n}$ and $L \nless L_{-}$, then

$$
L=\vee\left\{N_{1} \otimes \cdots \otimes N_{n}: N_{1} \otimes \cdots \otimes N_{n} \leqslant L\right\} .
$$

Proof: Suppose that $0 \neq x \in L$. Since $L \nless L_{-}, L_{-} \neq I_{1} \otimes \cdots \otimes I_{n}$. For any $0 \neq y \in L_{-}^{\perp}$, Lemma 1 shows that the rank-one operator $x y^{*} \in \operatorname{Alg}\left(\mathcal{L}_{1} \otimes \cdots \otimes \mathcal{L}_{n}\right)$. By Lemma 3, there exist $N_{i} \in \mathcal{L}_{i}(i=1, \ldots, n)$ such that $x \in N_{1} \otimes \cdots \otimes N_{n}$ and $y \in N_{1-}^{\perp} \otimes \cdots \otimes N_{n-}^{\perp}$. If $N_{1} \otimes \cdots \otimes N_{n} \nless L$, it follows from the definition of $\left(N_{1} \otimes \cdots \otimes N_{n}\right)_{-}$ that $L \leqslant\left(N_{1} \otimes \cdots \otimes N_{n}\right)_{-}$. By virtue of Lemma 2, we then have

$$
\begin{aligned}
L & \leqslant\left(N_{1} \otimes \cdots \otimes N_{n}\right)_{-} \\
& \leqslant\left(I_{1} \otimes \cdots \otimes N_{i} \otimes \cdots \otimes I_{n}\right)_{-} \\
& =I_{1} \otimes \cdots \otimes N_{i-} \otimes \cdots \otimes I_{n}
\end{aligned}
$$

and

$$
L^{\perp} \geqslant I_{1} \otimes \cdots \otimes N_{i-}^{\perp} \otimes \cdots \otimes I_{n}, \quad \text { for each } i .
$$

So $L^{\perp} \geqslant N_{1-}^{\perp} \otimes \cdots \otimes N_{n-}^{\perp}$ and we have shown that $y \in L^{\perp}$. Thus for any $y \in L_{-}^{\perp}$, we show that $y \in L^{\perp}$. This implies that $L_{-}^{\perp} \leqslant L^{\perp}$ and $L \leqslant L_{-}$. This contradicts our hypothesis. Hence $N_{1} \otimes \cdots \otimes N_{n} \leqslant L$ and for any $x \in L$,

$$
x \in \vee\left\{N_{1} \otimes \cdots \otimes N_{n}: N_{1} \otimes \cdots \otimes N_{n} \leqslant L\right\} .
$$

Thus

$$
L \leqslant \vee\left\{N_{1} \otimes \cdots \otimes N_{n}: N_{1} \otimes \cdots \otimes N_{n} \leqslant L\right\} .
$$

The converse inequality is obvious and this completes the proof.

Lemma 5. Let $x_{i}, y_{i} \in \mathcal{H}_{i}(i=1, \ldots, n)$. Then

$$
\left(x_{1} \otimes \cdots \otimes x_{n}\right)\left(y_{1} \otimes \cdots \otimes y_{n}\right)^{*}=\left(x_{1} y_{1}^{*}\right) \otimes \cdots \otimes\left(x_{n} y_{n}^{*}\right) .
$$

Proof: For any $z_{i} \in \mathcal{H}_{i}$, it follows from the definition that

$$
\begin{aligned}
{\left[( x _ { 1 } \otimes \cdots \otimes x _ { n } ) \left(y_{1} \otimes \cdots \otimes\right.\right.} & \left.\left.y_{n}\right)^{*}\right]\left(z_{1} \otimes \cdots \otimes z_{n}\right) \\
& =\left\langle z_{1} \otimes \cdots \otimes z_{n}, y_{1} \otimes \cdots \otimes y_{n}\right\rangle\left(x_{1} \otimes \cdots \otimes x_{n}\right) \\
& =\left\langle z_{1}, y_{1}\right\rangle \cdots\left\langle z_{n}, y_{n}\right\rangle\left(x_{1} \otimes \cdots \otimes x_{n}\right) \\
& =\left(\left\langle z_{1}, y_{1}\right\rangle x_{1}\right) \otimes \cdots \otimes\left(\left\langle z_{n}, y_{n}\right\rangle x_{n}\right) \\
& =\left[\left(x_{1} y_{1}^{*}\right) z_{1}\right] \otimes \cdots \otimes\left[\left(x_{n} y_{n}^{*}\right) z_{n}\right] \\
& =\left[\left(x_{1} y_{1}^{*}\right) \otimes \cdots \otimes\left(x_{n} y_{n}^{*}\right)\right]\left(z_{1} \otimes \cdots \otimes z_{n}\right) .
\end{aligned}
$$


Since the linear span of simple tensors is everywhere dense in $\mathcal{H}_{1} \otimes \cdots \otimes \mathcal{H}_{n}$, so

$$
\left(x_{1} \otimes \cdots \otimes x_{n}\right)\left(y_{1} \otimes \cdots \otimes y_{n}\right)^{*}=\left(x_{1} y_{1}^{*}\right) \otimes \cdots \otimes\left(x_{n} y_{n}^{*}\right)
$$

TheOREM 6. Suppose that $\mathcal{L}_{i} \subseteq \mathcal{B}\left(\mathcal{H}_{i}\right)(i=1, \ldots, n)$ are subspace lattices and $\operatorname{Alg}\left(\mathcal{L}_{1} \otimes \cdots \otimes \mathcal{L}_{n}\right)$ is weakly generated by rank-one operators in itself. Then

$$
\operatorname{Alg}\left(\mathcal{L}_{1} \otimes \cdots \otimes \mathcal{L}_{n}\right)=\operatorname{Alg} \mathcal{L}_{1} \otimes_{w} \cdots \otimes_{w} \operatorname{Alg} \mathcal{L}_{n}
$$

Proof: Each of the operators which generate $\operatorname{Alg} \mathcal{L}_{1} \otimes_{w} \cdots \otimes_{w} \operatorname{Alg} \mathcal{L}_{n}$ leaves invariant each of the projections which generate $\mathcal{L}_{1} \otimes \cdots \otimes \mathcal{L}_{n}$; therefore

$$
\operatorname{Alg}\left(\mathcal{L}_{1} \otimes \cdots \otimes \mathcal{L}_{n}\right) \supseteq \operatorname{Alg} \mathcal{L}_{1} \otimes_{w} \cdots \otimes_{w} \operatorname{Alg} \mathcal{L}_{n}
$$

It remains to show that $\operatorname{Alg}\left(\mathcal{L}_{1} \otimes \cdots \otimes \mathcal{L}_{n}\right) \subseteq \operatorname{Alg} \mathcal{L}_{1} \otimes_{w} \cdots \otimes_{w} \operatorname{Alg} \mathcal{L}_{n}$. Since $\operatorname{Alg}\left(\mathcal{L}_{1} \otimes \cdots \otimes \mathcal{L}_{n}\right)$ is weakly generated by rank-one operators in itself, it suffices to show that each rank-one operator in $\operatorname{Alg}\left(\mathcal{L}_{1} \otimes \cdots \otimes \mathcal{L}_{n}\right)$ belongs to $\operatorname{Alg} \mathcal{L}_{1} \otimes_{w} \cdots \otimes_{w} \operatorname{Alg} \mathcal{L}_{n}$. Now for any $N_{i} \in \mathcal{L}_{i}$ and $x_{i}, y_{i} \in \mathcal{H}_{i}$, we have that

$$
\begin{aligned}
\left(N_{1} \otimes \cdots \otimes\right. & \left.N_{n}\right)\left[\left(x_{1} \otimes \cdots \otimes x_{n}\right)\left(y_{1} \otimes \cdots \otimes y_{n}\right)^{*}\right]\left(N_{1-}^{\perp} \otimes \cdots \otimes N_{n-}^{\perp}\right) \\
& =\left(N_{1} \otimes \cdots \otimes N_{n}\right)\left[\left(x_{1} y_{1}^{*}\right) \otimes \cdots \otimes\left(x_{n} y_{n}^{*}\right)\right]\left(N_{1-}^{\perp} \otimes \cdots \otimes N_{n-}^{\perp}\right) \\
& =N_{1}\left(x_{1} y_{1}^{*}\right) N_{1-}^{\perp} \otimes \cdots \otimes N_{n}\left(x_{n} y_{n}^{*}\right) N_{n-}^{\perp} \\
& \in A \lg \mathcal{L}_{1} \otimes_{w} \cdots \otimes{ }_{w} \operatorname{Alg} \mathcal{L}_{n} .
\end{aligned}
$$

For any rank-one operator $z w^{*} \in \operatorname{Alg}\left(\mathcal{L}_{1} \otimes \cdots \otimes \mathcal{L}_{n}\right)$, it follows from Lemma 3 that there exist $N_{i} \in \mathcal{L}_{i}(i=1, \ldots, n)$ such that $z \in N_{1} \otimes \cdots \otimes N_{n}$ and $w \in N_{1-}^{\perp} \otimes \cdots \otimes N_{n-}^{\perp}$. Since $z, w \in \mathcal{H}_{1} \otimes \cdots \otimes \mathcal{H}_{n}$, there exist sequences $\left\{z_{m}\right\}$ and $\left\{w_{m}\right\}$ such that

$$
z_{m} \stackrel{\|\cdot\|}{\rightarrow} z \quad \text { and } \quad w_{m} \stackrel{\|\cdot\|}{\rightarrow} w
$$

where $z_{m}, w_{m}$ are finite linear combinations of simple tensors. It is routine to show that

$$
\left(N_{1} \otimes \cdots \otimes N_{n}\right)\left(z_{m} w_{m}^{*}\right)\left(N_{1-}^{1} \otimes \cdots \otimes N_{n-}^{1}\right) \stackrel{\|\cdot\|}{\rightarrow}\left(N_{1} \otimes \cdots \otimes N_{n}\right)\left(z w^{*}\right)\left(N_{1-}^{\perp} \otimes \cdots \otimes N_{n-}^{1}\right)=z w^{*} .
$$

The preceding pragragh shows that

$$
\left(N_{1} \otimes \cdots \otimes N_{n}\right)\left(z_{m} w_{m}^{*}\right)\left(N_{1-}^{\perp} \otimes \cdots \otimes N_{n-}^{\perp}\right) \in \operatorname{Alg} \mathcal{L}_{1} \otimes_{w} \cdots \otimes_{w} \operatorname{Alg} \mathcal{L}_{n}
$$

so $z w^{*} \in \operatorname{Alg} \mathcal{L}_{1} \otimes_{w} \cdots \otimes_{w} \operatorname{Alg} \mathcal{L}_{n}$. This completes the proof.

Corollary 7 . Let $\mathcal{L}_{i} \subseteq \mathcal{B}\left(\mathcal{H}_{i}\right)(i=1, \ldots, n)$ be subspace lattices. If $\operatorname{Alg}\left(\mathcal{L}_{1}\right.$ $\left.\otimes \cdots \otimes \mathcal{L}_{n-1}\right)$ is weakly generated by rank-one operators in itself, then

$$
\operatorname{Alg}\left(\mathcal{L}_{1} \otimes \cdots \otimes \mathcal{L}_{n}\right)=\operatorname{Alg} \mathcal{L}_{1} \otimes_{w} \cdots \otimes_{w} \operatorname{Alg} \mathcal{L}_{n}
$$


Proof: It follows from [4, Proposition 1.1 and Theorem 2.1] that

$$
\operatorname{Alg}\left(\mathcal{L}_{1} \otimes \cdots \otimes \mathcal{L}_{n}\right)=\operatorname{Alg}\left(\mathcal{L}_{1} \otimes \cdots \otimes \mathcal{L}_{n-1}\right) \otimes_{w} \operatorname{Alg} \mathcal{L}_{n}
$$

By virtue of Theorem 6 , we obtain that

$$
\operatorname{Alg}\left(\mathcal{L}_{1} \otimes \cdots \otimes \mathcal{L}_{n}\right)=\operatorname{Alg} \mathcal{L}_{1} \otimes_{w} \cdots \otimes_{w} \operatorname{Alg} \mathcal{L}_{n}
$$

The following corollary is one of the main results in [3].

Corollary 8. ([3, Theorem 17].) Let $\mathcal{L}_{i} \subseteq \mathcal{B}\left(\mathcal{H}_{i}\right)(i=1, \ldots, n)$ be completely distributive commutative subspace lattices. Then

$$
\operatorname{Alg}\left(\mathcal{L}_{1} \otimes \cdots \otimes \mathcal{L}_{n}\right)=\operatorname{Alg} \mathcal{L}_{1} \otimes_{w} \cdots \otimes_{w} \operatorname{Alg} \mathcal{L}_{n}
$$

Proof: It follows from [3, Theorem 10] that $\mathcal{L}_{1} \otimes \cdots \otimes \mathcal{L}_{n}$ is a completely distributive commutative subspace lattice. Thus, by virtue of [5, Theorem 3], $\operatorname{Alg}\left(\mathcal{L}_{1} \otimes \cdots \otimes \mathcal{L}_{n}\right)$ is weakly generated by the rank-one operators in itself. So the corollary follows from Theorem 6.

Corollary 9. ([1, Theorem 2.6].) Let $\mathcal{N}_{i}(i=1, \ldots, n)$ be nests. Then

$$
\operatorname{Alg}\left(\mathcal{N}_{1} \otimes \cdots \otimes \mathcal{N}_{n}\right)=\operatorname{Alg} \mathcal{N}_{1} \otimes_{w} \cdots \otimes_{w} \operatorname{Alg} \mathcal{N}_{n}
$$

If $\mathcal{L}$ is a subspace lattice, let $\mathcal{R}(\mathcal{L})$ denote the linear span of rank-one operators in Alg $\mathcal{L}$ and $\overline{\mathcal{R}(\mathcal{L})}$ the norm closure of $\mathcal{R}(\mathcal{L})$. If $\mathcal{S}_{i} \subseteq \mathcal{B}\left(\mathcal{H}_{i}\right)(i=1, \ldots, n)$ are subspaces, $\mathcal{S}_{1} \bar{\otimes} \cdots \bar{\otimes} \mathcal{S}_{n}$ denotes the norm closure of $\mathcal{S}_{1} \otimes \cdots \otimes \mathcal{S}_{n}$.

Proposition 10. Let $\mathcal{L}_{i} \subseteq \mathcal{B}\left(\mathcal{H}_{i}\right)(i=1, \ldots, n)$ be subspace lattices. Then

$$
\overline{\mathcal{R}\left(\mathcal{L}_{1} \otimes \cdots \otimes \mathcal{L}_{n}\right)}=\mathcal{R}\left(\mathcal{L}_{1}\right) \bar{\otimes} \cdots \bar{\otimes} \mathcal{R}\left(\mathcal{L}_{n}\right)
$$

PROOF: The result is essentially implied in the proof of Theorem 6.

Note that in Proposition 10 we do not need the hypothesis that $\operatorname{Alg}\left(\mathcal{L}_{1} \otimes \cdots \otimes \mathcal{L}_{n}\right)$ is weakly generated by rank-one operators in itself.

\section{REFERENCES}

[1] F. Gilfeather, A. Hopenwasser and D. Larson, 'Reflexive algebras with finite width lattices: tensor products, cohomology, compact perturbation', J. Funct. Anal. 55 (1984), 176-199.

[2] A. Hopenwasser and J. Kraus, 'Tensor products of reflexive algebras II', J. London Math. Soc. 228 (1983), 359-362.

[3] A. Hopenwasser, C. Laurie and R. Moore, 'Reflexive algebras with completely distributive subspace lattices', J. Operator Theory 11 (1984), 91-108.

[4] J. Kraus, 'Tensor products of reflexive algebras', J. London Math. Soc. 228 (1983), $350-358$. 
[5] C. Laurie and W.E. Longstaff, 'A note on rank-one operators in reflexive algebras', Proc. Amer. Math. Soc. 89 (1983), 293-297.

[6] W.E. Longstaff, 'Strongly reflexive lattices', J. London Math.Soc. 211 (1975), 491-498.

Institute of Mathematics

Fudan University

Shanghai 200433

People's Republic of China

e-mail: dzhe8@mail.china.com 